\title{
DISPLACEMENT ANALYSIS OF THE MEMS DEVICE
}

\author{
Ishak Ertugrul * \\ Mus Alparslan University, Faculty of Technical Science, Department of Mechatronics, \\ Mus, 49100, Turkey
}

Received 26.05.2020

Accepted 27.08.2020

\begin{abstract}
In this study, the displacement analysis of the microelectromechanical system (MEMS) device was performed. The current passing through the microdevice radiates heat energy as it pushes the device to the desired distance through thermal expansion. The amount of expansion varies depending on the current flowing through the device. With the designed model, the amount of current required for the displacement of the MEMS device is determined. In addition, the displacements produced in the microdevice for different metallic materials (silver and gold) and input potentials $(0.4 \mathrm{~V}, 0.8 \mathrm{~V}$, and 1.2 V) were calculated. These types of materials are frequently preferred in MEMS technology due to their high conductivity. Increasing the voltage value as a result of the analysis studies increased the displacement of the materials. When $1.2 \mathrm{~V}$ voltage is applied, the highest displacement values for silver and gold are; $6.45 \mu \mathrm{m}, 4.32 \mu \mathrm{m}$, respectively. According to the results, the silver material showed a significant displacement compared to gold material.
\end{abstract}

Keywords: MEMS; displacement; metallic materials; current.

${ }^{*}$ Corresponding author: Ishak Ertugrul, i.ertugrul@alparslan.edu.tr 


\section{Introduction}

Microelectromechanical systems (MEMS) are used to create miniature, highly sensitive sensors and actuators that can collect non-electronic information from the physical world such as temperature, barometric pressure, relative humidity, acceleration, vibration [1,2]. This analog information can be digitized and transmitted to a microcontroller for signal processing. When a complex signal analysis is required, such as the extraction of temporal patterns, the signal can be transmitted to a radio frequency high-quality computer [3, 4].

MEMS is an integrated electromechanical system in which the specification size and operating range of the components are on a micro-scale. Unlike traditional mechanical processing, the manufacturing of the MEMS device uses the semiconductor manufacturing process that includes surface micro-processing and batch microprocessing, which can be compatible with an integrated circuit [5]. These devices or systems are capable of sensing, controlling, actuating, and creating macro-scale effects on a micro-scale [6-8].

Thanks to the ever-increasing process technology, a large number of complex microstructural and functional modules are available today, leading to better and more optimized performances of the devices to be developed [9]. Electrostatic driven MEMS devices have the advantages of fast response, lower power consumption, and integrated circuit standard process compatibility [10].

Compared to LSI devices that only deal with electrical signals, MEMS devices are concerned with the conversion and integration of a wide variety of signal types such as physical (electrical, mechanical, thermal, optical, etc.), chemical, and biological signals [11]. Overall, silicon MEMS technology offers the possibility of low-cost, highperformance, and miniature multi-functional integrated devices for use in a wide variety of consumer and industrial applications such as the automotive, biomedical and telecommunications industries, defense, and the like [12, 13].

There are three main sources of stresses in thin films and multilayered plates: structural, thermal, and mechanical. Structural and thermal stresses are generally called residual stresses. When the film and coating material (or layers in a multilayer layer) have different thermal expansion coefficients (CTE), stresses occur due to temperature changes. These stress values can be very large (a few GPa) for some systems and often provide actuating power for mechanical failure. When the temperature differs from the accumulation temperature, the remaining voltages include both structural and thermal additives [14-18].

The induced stress loads the material and causes the structure through which the current passes to displace. Thermal expansion depends on the amount of current flowing through it and the temperature emitted. The application of the MEMS device is to move the device to the desired distance by transmitting an appropriate amount of current through the conductive layers and causing its displacement by thermal expansion [19]. This model is designed with the COMSOL program. This program was chosen to model and simulate the microdevice made of different metal materials at different potentials.

In this article, the design and displacement analysis of the MEMS based microdevice has been performed. In the proposed model, displacements as a result of applied electric potentials are analyzed. The displacements occurring in the device for different potentials $(0.4 \mathrm{~V}, 0.8 \mathrm{~V}$, and $1.2 \mathrm{~V})$ and materials (silver and gold) were investigated. 


\section{Experimental}

\section{Design Conditions}

MEMS based microdevice designed in COMSOL program has a length of $200 \mu \mathrm{m}$, a height of $30 \mu \mathrm{m}$, and a width of $40 \mu \mathrm{m}$. Silver and gold metallic materials are used in the microdevice. With this design, the displacement analyzes of the materials were carried out. Support points at both ends of the microdevice are firmly connected to a substrate. The electric potential at $0.4 \mathrm{~V}, 0.8 \mathrm{~V}$, and $1.2 \mathrm{~V}$ voltage is applied through the pads between the support points. Thermally-induced stress loads the material and causes it to displace. As a first approximation, we can assume that the electrical conductivity is constant. However, the resistance of a conductor increases with temperature. The relationship between resistance and temperature is approximately linear over a wide temperature range.

The geometric structure of the microdevice designed as 3D (3-dimensional plane) using COMSOL is shown in Figure 1. Metallic material has been added to the proposed geometric structure. While browsing all available databases, specific material is selected, and properties are assigned to the selected material. Silver (Ag) and gold (Au) were selected from the material scanner for this study. These materials are used as functional materials in the MEMS field due to their important physical and electrical properties. The physical properties of the materials used in the analysis phase are given in Table 1.

Table 1. Physical properties of the materials.

\begin{tabular}{llll}
\hline $\begin{array}{l}\text { Parameter } \\
\text { Thermal Expansion }\end{array}$ & Silver & Gold & Unit \\
$\begin{array}{l}\text { Coefficient } \\
\text { Density }\end{array}$ & $18.9 \cdot 10^{-6}$ & $14.2 \cdot 10^{-6}$ & $1 / \mathrm{K}$ \\
Thermal Conductivity & 429 & 19300 & $\mathrm{~kg} / \mathrm{m}^{3}$ \\
Young's Modulus & $83 \cdot 10^{9}$ & 317 & $\mathrm{~W} /(\mathrm{m} \cdot \mathrm{K})$ \\
Poisson Ratio & 0.37 & $70 \cdot 10^{9}$ & $\mathrm{~Pa}$ \\
\hline
\end{tabular}

This study demonstrates the ability to combine thermal, electrical, and structural analysis into a single model. With the application, movement is provided by passing a current through a microdevice; heat is produced by current, and the rise in temperature leads to displacement through thermal expansion. The model estimates how much current and temperature increase is required to displace the microdevice.

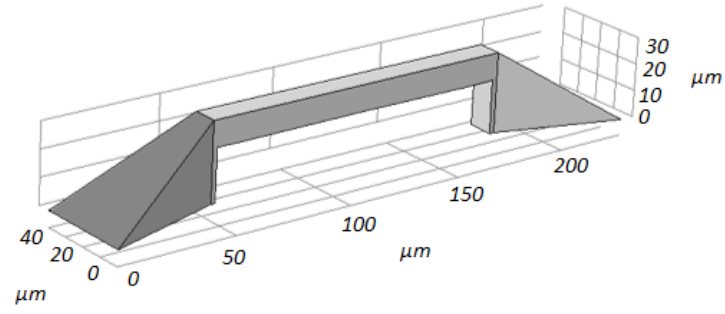

Fig. 1. The designed microdevice. 


\section{Results and Discussions}

\section{Simulation}

The simulation process was carried out in two stages. Initially, the silver microdevice was designed, and its displacement was carried out for three different input potentials. In the second stage, displacements were performed for the gold device instead of gold material for similar input potentials. The support points of the microdevice at both ends are fixed on a substrate, and an electrical potential is applied through the pads. The applied electric potential induces an electric current in the microdevice; the current passing through the structure causes some slowdown in the flow of electrons where energy is emitted in the form of heat. This generated heat induces thermal stress on the device and causes it to move. The displacement amount in the microdevice when the electrical potential of $0.4 \mathrm{~V}, 0.8 \mathrm{~V}$, and $1.2 \mathrm{~V}$ are applied between the support points of the microdevice made of silver material, respectively, are shown in Fig. 2.



(a)

(b)

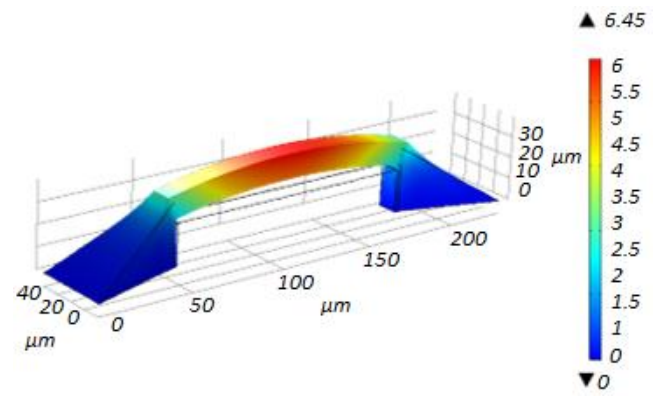

(c)

Fig. 2. Displacement data in silver when voltage of: (a) $0.4 \mathrm{~V}$ is applied (b) $0.8 \mathrm{~V}$ is applied (c) $1.2 \mathrm{~V}$ is applied.

The displacement amount in the microdevice when the electrical potential of 0.4 $\mathrm{V}, 0.8 \mathrm{~V}$, and $1.2 \mathrm{~V}$ are applied between the support points of the microdevice made of gold material, respectively, are shown in Fig. 3. 


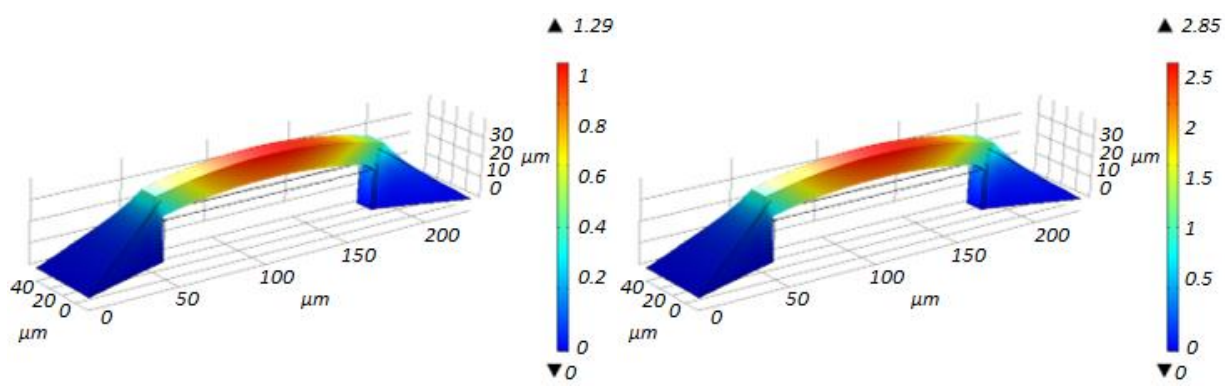

(a)

(b)

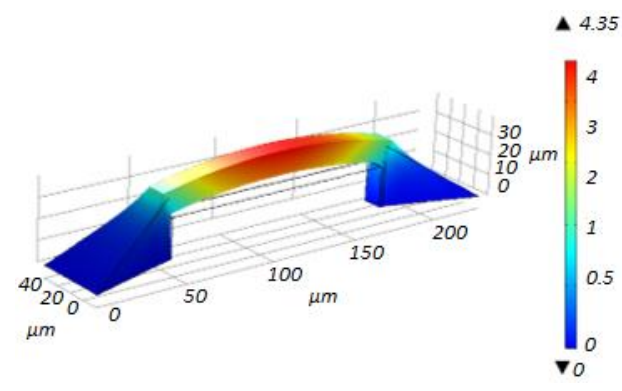

(c)

Fig. 3. Displacement data in gold when voltage of: (a) $0.4 \mathrm{~V}$ is applied, (b) $0.8 \mathrm{~V}$ is applied, (c) $1.2 \mathrm{~V}$ is applied.

Results

The model was analyzed by applying gold and silver materials to the microdevice. Analysis operations were carried out with variable electrical potentials $(0.4 \mathrm{~V}, 0.8 \mathrm{~V}$, and $1.2 \mathrm{~V}$ ) applied to each material. Firstly, an observation was made on the amount of displacement that occurs by passing a current through the device. The following table (Table 2) shows the amount of displacement data that occur in metal materials at three different input potentials. Gold material showed low displacement compared to silver owing to its low expansion coefficient.

Table 2. Displacement at different voltages.

\begin{tabular}{llll}
\hline Voltage, $V$ & 0.4 & 0.8 & 1.2 \\
Silver, displacement, $\mu \mathrm{m}$ & 2.18 & 4.19 & 6.45 \\
Gold, displacement, $\mu \mathrm{m}$ & 1.29 & 2.85 & 4.35 \\
\hline
\end{tabular}

Analysis of these results showed that silver materials give a significant displacement for the proposed geometry owing to the high value of the thermal expansion coefficient of the metallic microdevice (Table 2). The proposed geometry will be useful in the fabrication of MEMS devices to produce the desired displacements of all microscale device structures through the simple phenomenon of thermal expansion associated with the current through the microdevice. 


\section{Conclusions}

In this study, MEMS based microdevice is designed using COMSOL software. The current flowing through the device caused displacement through thermal expansion. Combining thermal, electrical and structural analysis made it possible to estimate the current and temperature rise required to displace the microdevice

The displacement amounts produced for the microdevice designed from two different materials were realized with different input potentials $(0.4 \mathrm{~V}, 0.8 \mathrm{~V}$, and $1.2 \mathrm{~V})$. According to the results, the microdevice designed with silver material showed significant displacement for the proposed geometry compared to other materials. The highest displacement was obtained as 6.45 by applying $1.2 \mathrm{~V}$ voltage to the silver material. The lowest displacement was obtained as 1.29 by applying $0.4 \mathrm{~V}$ voltage to gold material.

\section{References}

[1] R. Crescenzi, M. Balucani, N.P. Belfiore: Journal of Micromechanics and Microengineering, 28 (2018) 154-161.

[2] M.K. Mishra, V. Dubey, P.M. Mishra, I. Khan: Journal of Engineering Research and Reports, 5 (2019) 1-24.

[3] R. Lu, M. Li, Y. Yang: Journal of Microelectromechanical Systems, 28 (2019) 209-218.

[4] H. Singh, J. Malhotra: Trans Electr Electron Mater (TEEM), 18 (2017) 16-20.

[5] H. Madinei, H. Khodaparast, M.I. Friswell: Energy, 149 (2018) 990-999.

[6] O. Ulkir: Materials Research Express, 7 (2020) 075015.

[7] S. Nihtianov, A. Luque, Editors: Smart Sensors and MEMS, $2^{\text {nd }}$ edition, Woodhead Publishing, 2018, 28-36.

[8] T. L. Narayana, K. G. Sravani, K. S. Rao: Cogent Engineering, 4 (2017) 1363356.

[9] H. Jiang, M. Huang, Y. Yu, X. Tian, X. Zhao: Sensors, 18 (2018) 94.

[10] M. Pallay, R. N. Miles, S. Towfighian: IEEE Transactions on Industrial Electronics, 67 (2020) 9833 - 9840.

[11] Y. Mita, E. Lebrasseur, Y. Okamoto, F. Marty, R. Setoguchi, K. Yamada: Japanese Journal of Applied Physics, 56 (2017) 06GA03.

[12] H. O. Ali: Transactions of the IMF, 95 (2017) 290-296.

[13] M. R. Solouk, M. H. Shojaeefard, M. Dahmardeh: Mechanical Systems and Signal Processing, 128 (2019) 389-404.

[14] J. Rodriguez, D.D. Gerrard, G. M. Glaze, S. Chandorkar, L. Comenecia, Y. Chen, In: IEEE Sensors, 2017, 17452390.

[15] U. Brand, S. Gao, W. Engl, T. Sulzbach, S. W. Stahl, L. F. Milles, Z. Li: Measurement Science and Technology, 28 (2017) 034010.

[16] M. Vutukuru, J. W. Christopher, C. Pollock, D. J. Bishop, A. K. Swan: Journal of Microelectromechanical Systems, 28 (2019) 550-557.

[17] K. Özsoy, B. Duman, D. İçkale Gültekin: SDU International Journal of Technological Science, 11 (2019) 201-210.

[18] M. Y. Kayacan, K. Özsoy, B. Duman, N. Yilmaz, M. C. Kayacan: Materials and Manufacturing Processes, 34 (2019) 1467-1475.

[19] H. A. Hassen, K. Sofiane, Z. Bilel, Z. Ihsen, K. Sondes: The Pan African Medical Journal, 33 (2019) 107.

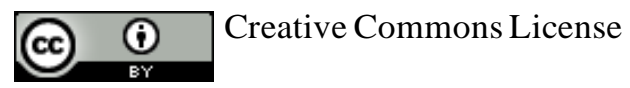

This work is licensed under a Creative Commons Attribution 4.0 International License. 